\title{
Finding proof negative: Tracing the empirical footprint of collective linguistic avoidance in historical American and British newspaper texts ${ }^{1}$
}

\author{
Donald S. MacQueen
}

\begin{abstract}
Linguistic avoidance - the conscious or unconscious non-use of a problematic language expression and/or the substitution of another - is difficult to demonstrate empirically in historical terms, as it constitutes a negative action that leaves no positive trace. However, all hope is not lost for the empiricist: the very conspicuousness of the problematic expression's absence can in fact be measured across a time period. A perhaps unique case in point in English is the avoidance of number-word expressions such as five millions of individuals and, alternatively, five million individuals precisely at the point in history when it became unclear which of the two expressions was the correct one, i.e. when usage was shifting. At this point, newspapers in the US and the UK, following patterns that were similar but not contemporaneous, began to favor numerical expressions like 5,000,000 individuals. The timing of the shift corresponded to the time gap between US and UK newspapers in their respective gradual rejection of the now obsolescent (Obs) five millions of individuals in favor of the present-day English (PdE) construction five million individuals. Then, in both countries, newspapers largely discontinued the use of numerical expressions like 5,000,000 individuals when it had become clear that the PdE construction had prevailed. In other words, the numerical expression was conspicuously substituted to avoid choosing between the Obs and PdE versions of the numberword expression, and this avoidance left a clear empirical trace.
\end{abstract}

\section{Background}

The issue of avoidance arose unexpectedly in an in-depth study of the integration of the number word MILLION into the English system of number words. The main results of this study have been reported elsewhere (MacQueen 2004, especially 2010, 2014), namely that MILLION underwent a change in word class and 
function from being a noun heading a noun phrase (example 1) to being a (post-) determiner of the true head of the noun phrase (2), which is the noun being counted (people).

(1) It has about five millions of people... Daily Telegraph editorial, May 27, 1910 (CENE Corpus)

(2) ...organizations representing five million people...

Michael W. Fox, Superpigs and Wondercorn: The Brave New World of Biotechnology and Where It All May Lead, 1992 (Frown Corpus)

In other words, MILLION completed the leveling of the paradigm for number words in English, behaving, after having been an outlier for roughly five centuries, just like THOUSAND, HUNDRED, etc. With this change all number words in English assumed what can be called their natural function in an ontological perspective (Hurford 1987:196-199). This shift took place in American newspapers roughly one generation before it occurred in The Times (ibid.). The precise dating will emerge from the figures reproduced below.

\section{$2 \quad$ Material and method}

The main findings were based on digital searches of Proquest's Historical Newspapers Collection (for US newspapers) and Gale Cengage's The Times Digital Archive (for the leading UK newspaper of the period covered). At the time the study was undertaken (roughly 2003-2008), these databases contained tens of billions of datable words from 1851 to the present (Historical Newspapers) and from 1785 to the present (Times Digital Archive), and they have continued to grow in the last few years. ${ }^{2}$

The methodology necessitated by the proprietary nature of these collections is also outlined in detail in the above sources and need not be elaborated here. Suffice it to say that all the results are based on 'proxy incidence,' meaning that incidence was determined not by the number of occurrences of the search string per total number of words in the texts but rather by the number of articles containing at least one instance of the search string per total number of articles. For comparing US/UK newspapers across the time period at hand, the key parameter was simply the respective percentages of the total number of articles with at least one instance of the expression. 


\section{The unexpected question and its answer: Avoidance}

As all my interest was focused on the respective proportions of $O b s$ and $P d E$ expressions, I was surprised to note, in perusing my tables, that the actual number of articles with one instance of either of the respective expressions clearly declined, then rose, then declined again for a couple of decades - earlier decades in the US newspapers, later ones in the UK newspaper - despite the fact that both databases could be shown to have grown in volume at the same time.

Table 1 shows that the combined totals of $O b s$ and $P d E$ expressions in Historical Newspapers Collection varied considerably across the 1851-1910 period, with some odd drops and slow increases - especially in the $1880 \mathrm{~s}$ - in a steadily growing database.

Table 1: Combined totals for the number of articles containing at least one instance of $\mathrm{Obs}$ or PdE versions of two-ten million/s/ in Historical Newspapers Collection, 1851-1910 (from MacQueen 2010: 142)

\begin{tabular}{|l|l|l|l|l|l|l|l|l|l|}
\hline $\mathbf{1 8 5 1}$ & $\mathbf{1 8 5 2}$ & $\mathbf{1 8 5 3}$ & $\mathbf{1 8 5 4}$ & $\mathbf{1 8 5 5}$ & $\mathbf{1 8 5 6}$ & $\mathbf{1 8 5 7}$ & $\mathbf{1 8 5 8}$ & $\mathbf{1 8 5 9}$ & $\mathbf{1 8 6 0}$ \\
\hline 227 & 600 & 670 & 674 & 603 & 700 & 804 & 796 & 827 & 953 \\
\hline $\mathbf{1 8 6 1}$ & $\mathbf{1 8 6 2}$ & $\mathbf{1 8 6 3}$ & $\mathbf{1 8 6 4}$ & $\mathbf{1 8 6 5}$ & $\mathbf{1 8 6 6}$ & $\mathbf{1 8 6 7}$ & $\mathbf{1 8 6 8}$ & $\mathbf{1 8 6 9}$ & $\mathbf{1 8 7 0}$ \\
\hline 1111 & 857 & 808 & 933 & 1477 & 1313 & 1217 & 947 & 799 & 1026 \\
\hline $\mathbf{1 8 7 1}$ & $\mathbf{1 8 7 2}$ & $\mathbf{1 8 7 3}$ & $\mathbf{1 8 7 4}$ & $\mathbf{1 8 7 5}$ & $\mathbf{1 8 7 6}$ & $\mathbf{1 8 7 7}$ & $\mathbf{1 8 7 8}$ & $\mathbf{1 8 7 9}$ & $\mathbf{1 8 8 0}$ \\
\hline 1435 & 1645 & 1377 & 1002 & 1017 & 1075 & 749 & 1102 & 1025 & 811 \\
\hline $\mathbf{1 8 8 1}$ & $\mathbf{1 8 8 2}$ & $\mathbf{1 8 8 3}$ & $\mathbf{1 8 8 4}$ & $\mathbf{1 8 8 5}$ & $\mathbf{1 8 8 6}$ & $\mathbf{1 8 8 7}$ & $\mathbf{1 8 8 8}$ & $\mathbf{1 8 8 9}$ & $\mathbf{1 8 9 0}$ \\
\hline 940 & 972 & 903 & 779 & 968 & 726 & 789 & 785 & 1195 & 1862 \\
\hline $\mathbf{1 8 9 1}$ & $\mathbf{1 8 9 2}$ & $\mathbf{1 8 9 3}$ & $\mathbf{1 8 9 4}$ & $\mathbf{1 8 9 5}$ & $\mathbf{1 8 9 6}$ & $\mathbf{1 8 9 7}$ & $\mathbf{1 8 9 8}$ & $\mathbf{1 8 9 9}$ & $\mathbf{1 9 0 0}$ \\
\hline 1584 & 1323 & 1427 & 1391 & 1476 & 1492 & 1740 & 1615 & 1965 & 2361 \\
\hline $\mathbf{1 9 0 1}$ & $\mathbf{1 9 0 2}$ & $\mathbf{1 9 0 3}$ & $\mathbf{1 9 0 4}$ & $\mathbf{1 9 0 5}$ & $\mathbf{1 9 0 6}$ & $\mathbf{1 9 0 7}$ & $\mathbf{1 9 0 8}$ & $\mathbf{1 9 0 9}$ & $\mathbf{1 9 1 0}$ \\
\hline 2427 & 2560 & 2825 & 2645 & 2628 & 2987 & 3218 & 2370 & 2646 & 2786 \\
\hline
\end{tabular}

Table 2 shows the corresponding totals for The Times Digital Archive, though from forty years later. Note especially the long-term dip starting after the middle of the 1920s: 
Table 2: Combined totals for number of articles containing at least one instance of Obs or PdE versions of two-ten million/s/ in The Times Digital Archive, 1890-1950 [1890 total = 785] (from MacQueen 2010:142).

\begin{tabular}{|l|l|l|l|l|l|l|l|l|l|}
\hline $\mathbf{1 8 9 1}$ & $\mathbf{1 8 9 2}$ & $\mathbf{1 8 9 3}$ & $\mathbf{1 8 9 4}$ & $\mathbf{1 8 9 5}$ & $\mathbf{1 8 9 6}$ & $\mathbf{1 8 9 7}$ & $\mathbf{1 8 9 8}$ & $\mathbf{1 8 9 9}$ & $\mathbf{1 9 0 0}$ \\
\hline 912 & 760 & 738 & 665 & 462 & 545 & 553 & 445 & 565 & 474 \\
\hline $\mathbf{1 9 0 1}$ & $\mathbf{1 9 0 2}$ & $\mathbf{1 9 0 3}$ & $\mathbf{1 9 0 4}$ & $\mathbf{1 9 0 5}$ & $\mathbf{1 9 0 6}$ & $\mathbf{1 9 0 7}$ & $\mathbf{1 9 0 8}$ & $\mathbf{1 9 0 9}$ & $\mathbf{1 9 1 0}$ \\
\hline 463 & 449 & 563 & 492 & 474 & 474 & 705 & 663 & 768 & 624 \\
\hline $\mathbf{1 9 1 1}$ & $\mathbf{1 9 1 2}$ & $\mathbf{1 9 1 3}$ & $\mathbf{1 9 1 4}$ & $\mathbf{1 9 1 5}$ & $\mathbf{1 9 1 6}$ & $\mathbf{1 9 1 7}$ & $\mathbf{1 9 1 8}$ & $\mathbf{1 9 1 9}$ & $\mathbf{1 9 2 0}$ \\
\hline 660 & 699 & 566 & 485 & 506 & 403 & 462 & 474 & 754 & 608 \\
\hline $\mathbf{1 9 2 1}$ & $\mathbf{1 9 2 2}$ & $\mathbf{1 9 2 3}$ & $\mathbf{1 9 2 4}$ & $\mathbf{1 9 2 5}$ & $\mathbf{1 9 2 6}$ & $\mathbf{1 9 2 7}$ & $\mathbf{1 9 2 8}$ & $\mathbf{1 9 2 9}$ & $\mathbf{1 9 3 0}$ \\
\hline 580 & 839 & 915 & 542 & 544 & 387 & 299 & 297 & 219 & 139 \\
\hline $\mathbf{1 9 3 1}$ & $\mathbf{1 9 3 2}$ & $\mathbf{1 9 3 3}$ & $\mathbf{1 9 3 4}$ & $\mathbf{1 9 3 5}$ & $\mathbf{1 9 3 6}$ & $\mathbf{1 9 3 7}$ & $\mathbf{1 9 3 8}$ & $\mathbf{1 9 3 9}$ & $\mathbf{1 9 4 0}$ \\
\hline 117 & 118 & 120 & 151 & 159 & 120 & 162 & 135 & 177 & 98 \\
\hline $\mathbf{1 9 4 1}$ & $\mathbf{1 9 4 2}$ & $\mathbf{1 9 4 3}$ & $\mathbf{1 9 4 4}$ & $\mathbf{1 9 4 5}$ & $\mathbf{1 9 4 6}$ & $\mathbf{1 9 4 7}$ & $\mathbf{1 9 4 8}$ & $\mathbf{1 9 4 9}$ & $\mathbf{1 9 5 0}$ \\
\hline 79 & 79 & 86 & 98 & 98 & 122 & 107 & 79 & 95 & 184 \\
\hline
\end{tabular}

For a fuller picture, we need to see the number of articles per year in the respective collections across the same time periods. Figures 1 and 2 present these respective figures:

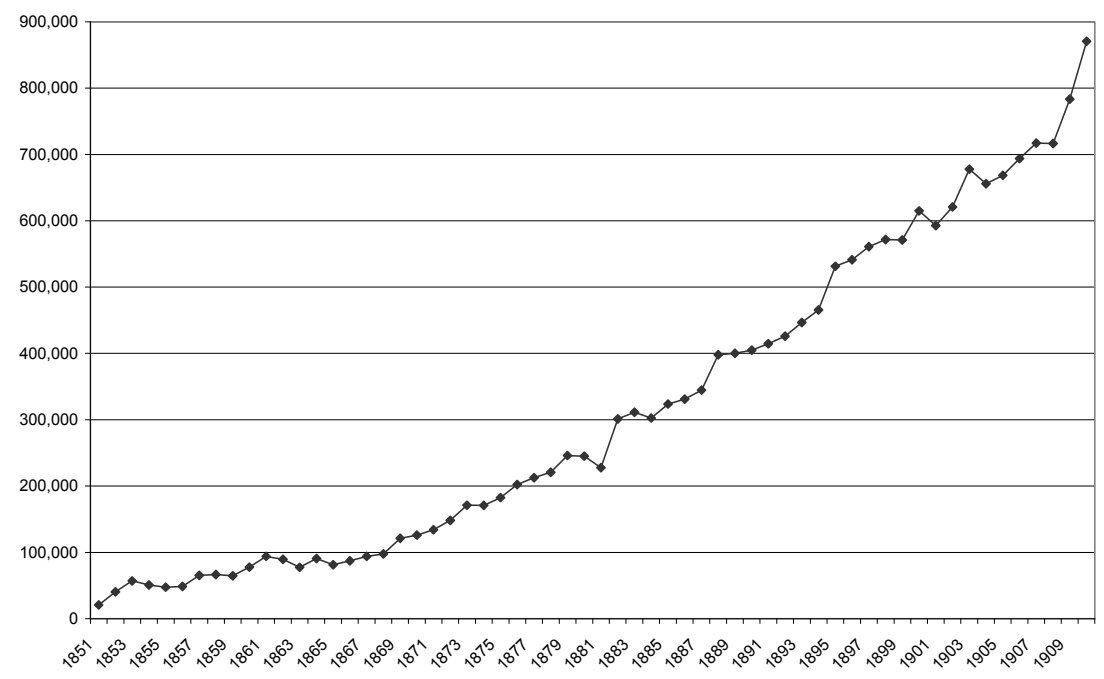

Figure 1: Number of articles containing at least one word in Historical Newspapers Collection, per year 1851-1910 (from MacQueen 2010:146) 


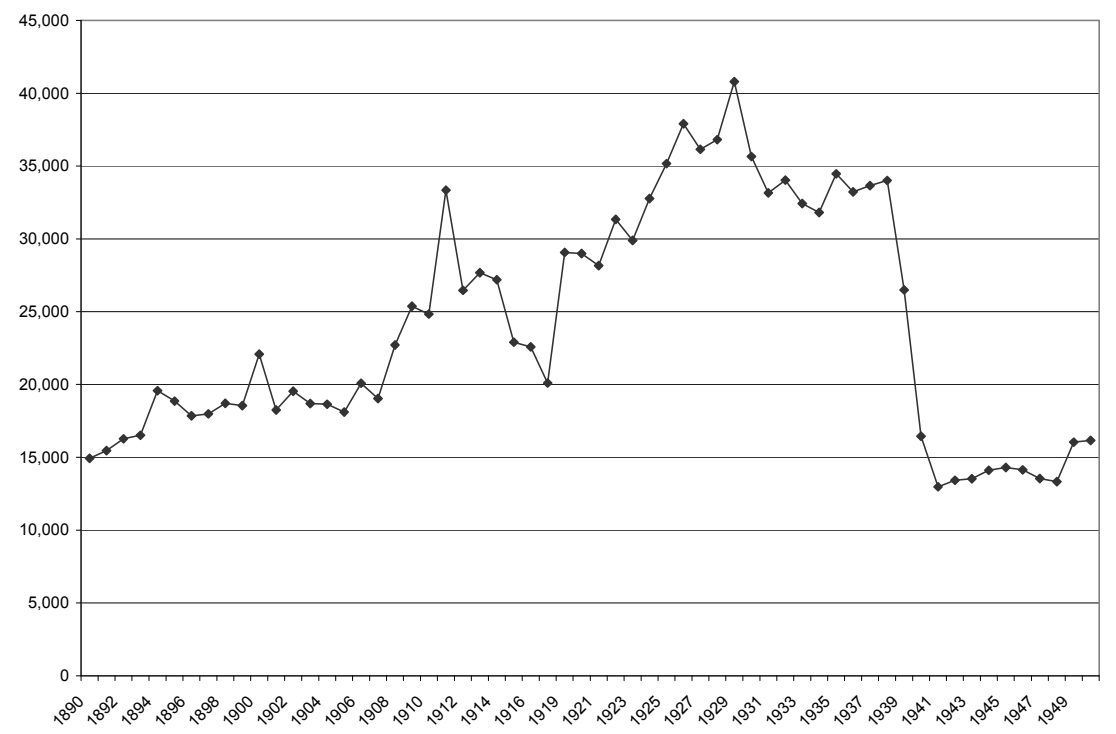

Figure 2: Number of articles containing the letter e in The Times Digital Archive per year 1890-1950 (from MacQueen 2010:147)

Figure 1 shows a steady rise in the number of articles included in the growing collection. Figure 2, based on the single newspaper The Times, displays a more erratic pattern, probably owing to the two world wars that so dramatically affected UK life. But for the period of immediate interest, there is a largely steady increase in the volume of the newspaper archive.

Figure 3 combines the information in Table 1 and Figure 1 for Historical Newspapers Collection by mapping the combined proxy incidence of Obs and $P d E$ expressions across the 1851-1910 period: 


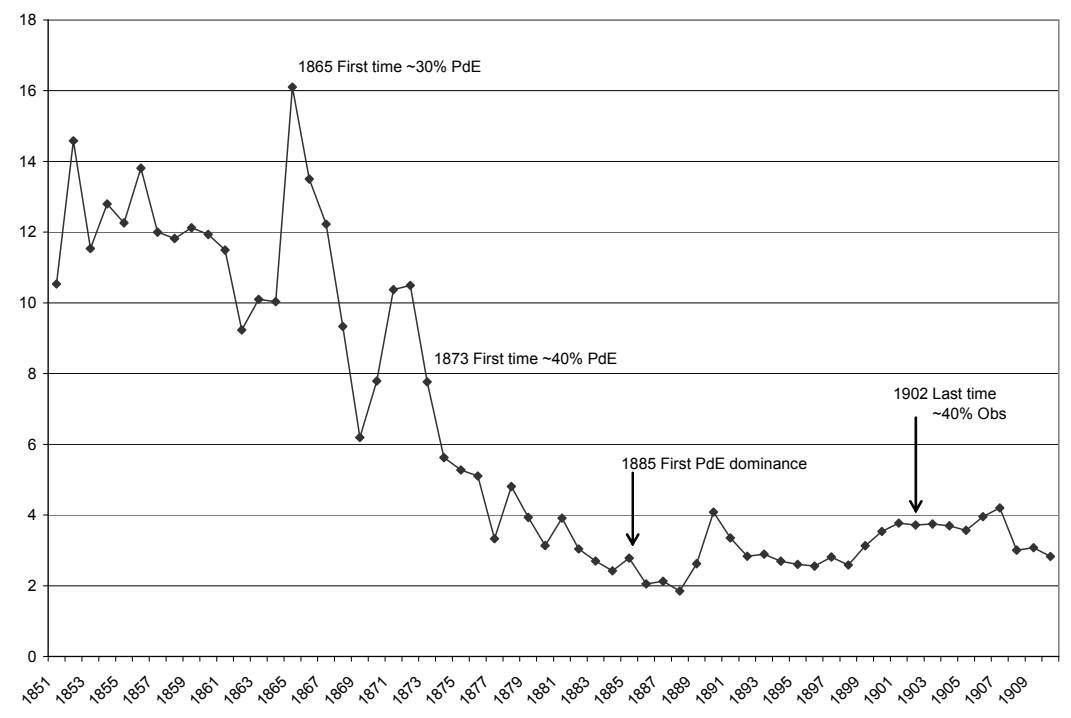

Figure 3: Proxy incidence of two-ten million/s/ in Historical Newspapers Collection per year 1851-1910 (from MacQueen 2010:149)

Figure 3 displays an occasionally erratic but basically steady decline in the proxy incidence of these number-word expressions. It also includes information about key years in the gradual shift from predominantly $O b s$ expressions to predominantly $P d E$ expressions: the first time $P d E$ represented about 30 percent of all (proxy) instances (1865); the first time $P d E$ expressions made up about 40 percent of all instances (1873); the first year dominated by the $P d E$ expression (1885); and the last time $O b s$ expressions reached about 40 percent of all instances (1902). (Proxy incidence figures for The Times Digital Archive 18901950 will be displayed as part of a more complex diagram in Figure 5 below.)

These unexpected drops in proxy incidence are puzzling, especially considering the tremendous growth in industrial output and the explosion of wartime statistics over the periods in question. The answer to the puzzle is to be sought in human nature and the natural reaction to language uncertainty: as these expressions in MILLION began to tip from $O b s$ to $P d E$ ascendancy, newspaper writers and editors began to leave it to their readers to decide how to say the phrases by encoding them in numerical form instead. Thus, two millions of individuals or 
two million individuals could be expressed as 2,000,000 individuals. Such a convenient avoidance strategy is rarely available to uncertain language users.

To test this hypothesis of a dynamic relationship between number-word and numerical expressions in MILLION, I searched for instances of 000,000 in the respective databases during the same relevant periods. Figure 4 plots the annual proxy incidence of number-word expressions against the annual proxy incidence of numerical expressions in the American Historical Newspapers Collection:

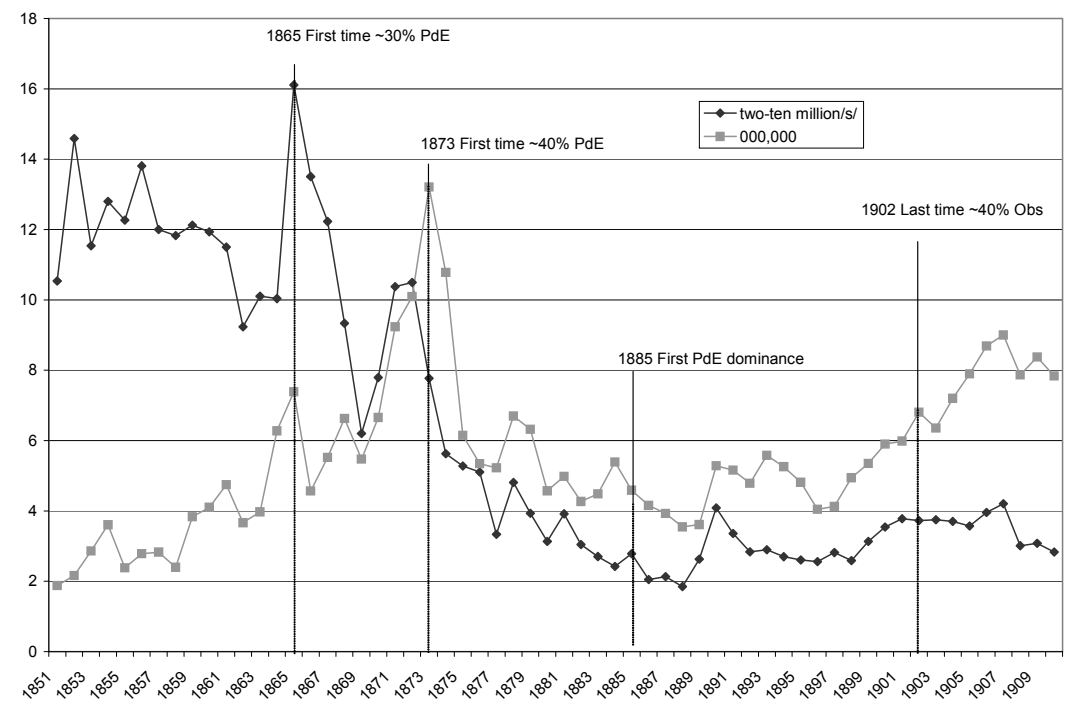

Figure 4: Comparative proxy incidence of two-ten million/s/ and 000,000 in Historical Newspaper Collection per year 1851-1910 (from MacQueen 2010:151)

Figure 4 reveals that numerical expressions were only about one sixth as frequent as their number-word equivalents at the beginning of the period. Both expressions peaked in the last three years of the American Civil War, especially in 1865 , which is the year when the $P d E$ expression first reached thirty percent of all instances. Nevertheless, numerical expressions are only about half as frequent as their number-word counterparts. There is another peak in 1872, when they are virtually equal in frequency. Then in 1873 , the year when the $P d E$ number-word expression first reached forty percent of all instances, numerical expressions continue to rise rapidly, while number-word expressions drop just as rapidly. From that point on, numerical expressions remain dominant for the rest 
of the period. Both types of expressions remain at a lower level from the late 1870 s through 1910 , with a widening gap between them starting in the $20^{\text {th }}$ century.

Figure 5 shows the same comparison for The Times Digital Archive across a century, 1851-1950, covering the periods of transition from Obs to PdE expressions in MILLION in both US newspapers and The Times:

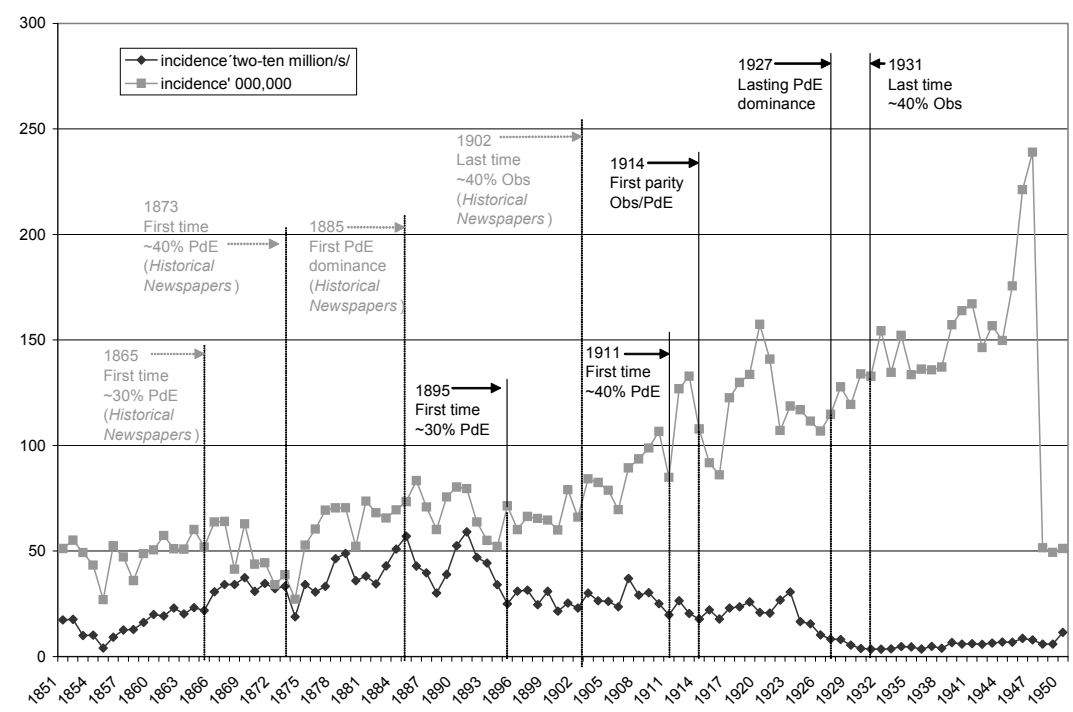

Figure 5: Comparative proxy incidence of two-ten million/s/ and 000,000 in The Times Digital Archive per year 1851-1950, with key years indicated (Historical Newspapers Collection key figures in gray) (from MacQueen 2010:153)

A salient feature of The Times data in Figure 5 is that numerical expressions dominate number-word expressions throughout the period. This contrasts with the strong dominance of number-word expressions in the US newspapers in the mid $19^{\text {th }}$ century, which was seen in Figure 4. Remarkably, the gap between the two lines, which had virtually closed through most of the 1870 s, starts to grow, albeit erratically, in 1895, the year when the proportion of $P d E$ usage reached the level of thirty percent for the first time in the UK. The gap continued to grow until 1947, by which time the proportion of the $P d E$ version of the number-word expression was above ninety percent. As the uncertainty had vanished, it might 
be supposed the editors of The Times decided to have $P d E$ number-word expressions replace numerical expressions in the newspaper's house style starting in 1948. The shift away from numerical expressions was drawn out over a much longer period in the multiple US newspapers included in Historical Newspapers Collection. This will be further examined below.

Thus, the increased substitution of numerical expressions in US newspapers and The Times seems to have been triggered in both cases by a roughly thirtypercent usage of the new $P d E$ form. This thirty- to forty-percent proportion of the new expression appears to have created a level of uncertainty among newspaper writers and editors that led them to avoid number-word expressions and substitute numerical expressions, leaving it to readers to unfold them into verbal expressions, whether silently in their minds or when reading the expression aloud.

\section{Decade-level overviews and endgame}

Having perused the data on the yearly level, we can now step back and look at the entire period of relevance for the respective American and British newspapers. Such a perspective also provides a clear picture of the endgame, that is, the respective periods when number-word expressions were no longer avoided and numerical expressions concomitantly declined in incidence. Figure 6 plots the proxy incidence per decade of number-word expressions, both $O b s$ and $P d E$ versions, against numerical expressions in million from the 1850 s to the (truncated) 2000s in Historical Newspapers Collection: 


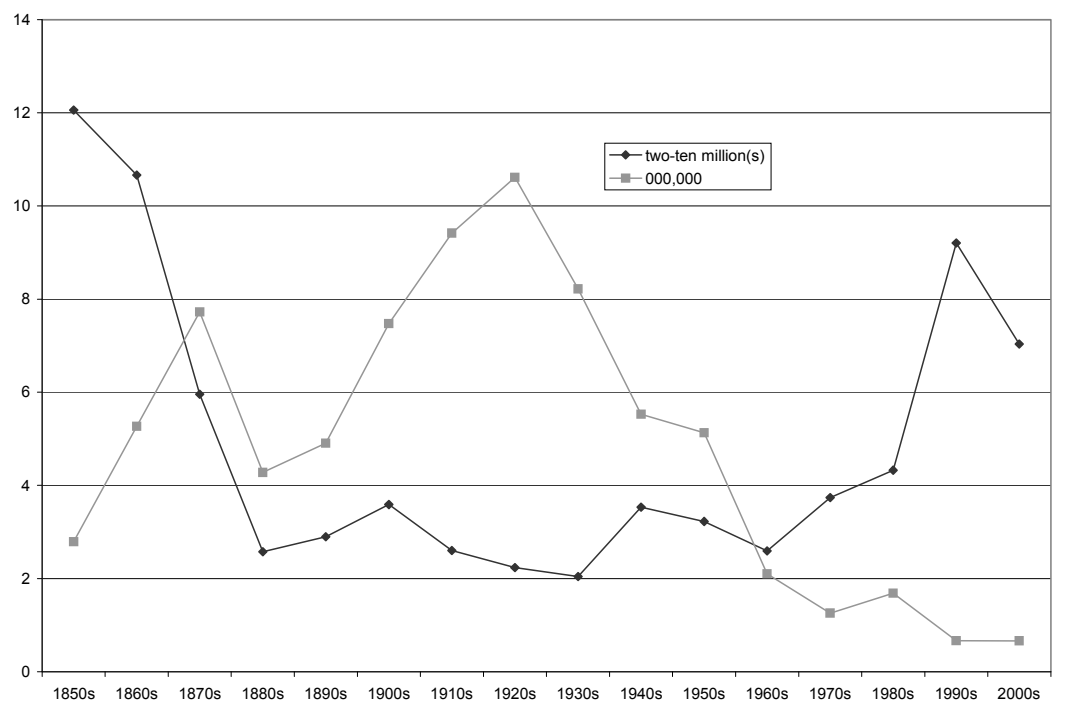

Figure 6: Comparative proxy incidence of two-ten million/s/ and 000,000 in Historical Newspapers Collection per decade, 1850s to 2000s (2000s truncated) (from MacQueen 2010:157)

Figure 7 similarly compares the proxy incidence per decade of the two versions of number-word expressions with numerical expressions from the 1850 s to the (truncated) 1980s in The Times Digital Archive: 


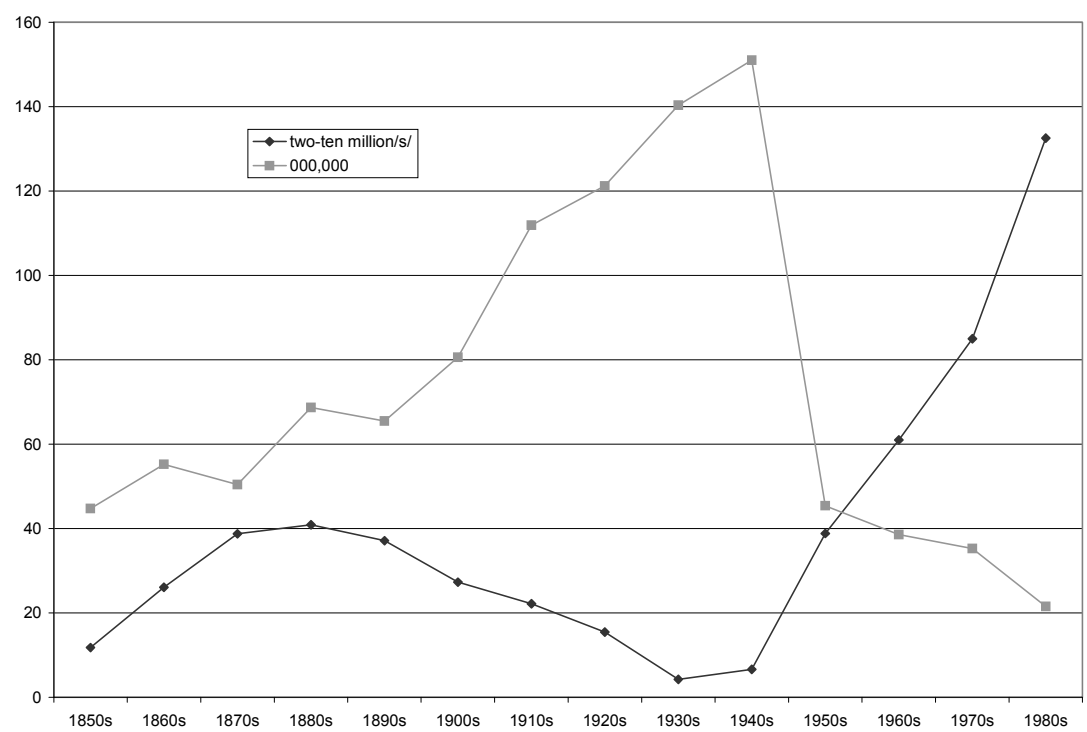

Figure 7: Comparative proxy incidence of two-ten million/s/ and 000,000 in The Times Digital Archive per decade, 1850s to 1980s (1980s truncated) (from MacQueen 2010: 158)

Figure 6 shows that the avoidance gap in the American newspapers lasted from the 1870 s to the 1950 s, with a major bulge between the 1900 s to the 1940 s. Figure 7 indicates that the gap in The Times stretched from the turn of the $20^{\text {th }}$ century to the 1940s, evincing a major bulge from the 1900s or 1910s through the 1940s. The decade of the peak differential was the 1920s for the American newspapers and the 1940s for The Times. The eventual shift to number-term dominance was more gradual in the US, quite abrupt in the UK newspaper, perhaps owing to a conscious decision to change the house style.

\section{$5 \quad$ An auxiliary reason for avoidance}

It should also be pointed out that in the $19^{\text {th }}$ and early $20^{\text {th }}$ centuries, using numerical expressions also had the advantage of saving column space for the newspapers. If we compare the length of the $O b s$ number word expression in (3) 
with the numerical expression in (4), we find that the latter requires considerably less column space than the former.

(3) two millions of

(4) $2,000,000$

More space would of course be saved for longer number words like seven or twelve millions of, and even more for compound number words like twenty-eight millions of. On the other hand, the space saved by substituting a numerical expression for the $P d E$ version of the word-number expression is minor in comparison with the same substitution for the $O b s$ version.

(5) two million

(6) $2,000,000$

Thus, saving column space became less of an argument for substituting numerical expressions once the $P d E$ number-word expression had become the standard. And using hybrid expressions, with numerals preceding the word million, saves a bit more column space.

(7) 2 million

(8) $2,000,000$

\section{Conclusion}

Whatever the influence of spatial economy might have been, it seems clear that the comparison of the proxy incidence of number-word expressions with numerical expressions by year and by decade provides, if not proof positive, then proof negative (modus tollens) of a pattern of linguistic avoidance in newspaper usage in the US and the UK, with the US pattern being largely reproduced roughly a generation later in the UK. While the phenomenon of avoidance is quite commonly referred to in psychological studies, to my knowledge it has yet to be addressed explicitly in linguistics outside of the context of taboo expressions. In the case presented above the empirical evidence strongly suggests that more than a century ago professional users of English began to avoid having to choose between number-word expressions like five millions of individuals or five million individuals because they were unsure which of them their readers would consider correct. Instead they turned to numerical expressions $(5,000,000$ individuals) and left it to the readers of their newspapers to unfold the numerical expression into whichever number-word expression they preferred. Many 
decades later, once it had become clear to everyone that the $P d E$ version, five million individuals (which is perfectly parallel to similar expressions in HUNDRED and THOUSAND, and which more economically and directly expresses the ontological sense of the expression) represented virtually the only choice, these professional users abandoned their numerical expressions and reverted to numberword equivalents.

Establishing the existence of a historical footprint in usage has been hugely facilitated by our access to very-large-scale electronic corpora and the possibility of searching them for specific variants of expressions. Of course, not all linguistic expressions that are undergoing change have a convenient alternate set of written characters - numerals and their attendant conventions - that can be pressed into service when doubts arise regarding correct usage, but it is intriguing to ponder what other avoidance footprints remain to be traced.

\section{Notes}

1. A version of this article was presented orally at the 10th Conference of the American Association of Corpus Linguistics, Georgia State University, Atlanta, October 7-9, 2011.

2. For the protean Proquest Newspaper Collection, see the Proquest webpage, specifically http:/www.proquest.com/libraries/academic/news-newspapers/ pq-hist-news.html (accessed January 19, 2015).

\section{References}

Hurford, James. 1984. Language and number: The emergence of a cognitive system. Oxford: Blackwell.

MacQueen, Donald. 2004. Developing methods for very-large-scale searches in Proquest Historical Newspapers Collection and Infotrac The Times Digital Archive. Journal of English Linguistics 32 (2): 124-143.

MacQueen, Donald. 2010. The integration of million into the English system of number words: A diachronic study. Frankfurt: Peter Lang.

MacQueen, Donald. 2014. American English influence on British English at the height of the British Empire: A case of cross-varietal easement. ICAME Journal 38: 105-113. 